\title{
Comparative integromics on Angiopoietin family members
}

\author{
YURIKO KATOH $^{1}$ and MASARU KATOH ${ }^{2}$ \\ ${ }^{1}$ M\&M Medical BioInformatics, Hongo 113-0033; ${ }^{2}$ Genetics and Cell Biology Section, \\ National Cancer Center Research Institute, Tokyo 104-0045, Japan
}

Received February 6, 2006; Accepted March 2, 2006

\begin{abstract}
Angiopoietin-1 (ANGPT1), Angiopoietin-4 (ANGPT4), VEGF, FGF2, FGF4, HGF, Ephrin, IL8 and CXCL12 (SFD1) are pro-angiogenic factors (angiogenic activators), while Angiopoietin-2 (ANGPT2), Angiostatin, Endostatin, Tumstatin, Canstatin, THBS1, THBS2, TNFSF15 (VEGI) and Vasohibin (VASH1) are anti-angiogenic factors (angiogenic inhibitors). ANGPT1 and ANGPT2 are ligands for TIE family receptor tyrosine kinases, TIE1 and TIE2 (TEK). Angiopoietin family consists of ANGPT1, ANGPT2, ANGPT4, ANGPTL1 (ANGPT3), ANGPTL2, ANGPTL3 (ANGPT5), ANGPTL4, ANGPTL5, ANGPTL6 and ANGPTL7. TCF/LEF binding sites within the promoter region of human Angiopoietin family members were searched for by using bioinformatics and human intelligence (Humint). Because four TCF/LEF-binding sites were identified within the human ANGPTL7 promoter, comparative genomics analyses on $A N G P T L 7$ orthologs were further performed. ANGPTL7 gene at human chromosome 1p36.22 was located within intron 28 of $F R A P 1$ gene encoding mTOR protein. Chimpanzee ANGPTL7 gene, consisting of five exons, was located within NW_101546.1 genome sequence. Chimpanzee ANGPTL7 showed $99.4 \%$ and $86.1 \%$ total-amino-acid identity with human ANGPTL7 and mouse Angptl7, respectively. Human ANGPTL7 mRNA was expressed in neural tissues, keratoconus cornea, trabecular meshwork, melanotic melanoma and uterus endometrial cancer, while mouse Angptl7 mRNA was expressed in four-cell embryo, synovial fibroblasts, thymus, uterus and testis. Four TCF/LEF-binding sites within human ANGPTL7 promoter were conserved in chimpanzee ANGPTL7 promoter; however, only an unrelated TCF/LEF-binding site occurred in mouse and rat Angptl7 promoters. Human ANGPTL7, characterized as potent target gene of WNT/ $\beta$-catenin signaling pathway, is a pharmacogenomics target in the fields of oncology and regenerative medicine.
\end{abstract}

Correspondence to: Dr Masaru Katoh, Genetics and Cell Biology Section, National Cancer Center Research Institute, 5-1-1 Tsukiji, Chuo-ku, Tokyo 104-0045, Japan

E-mail: mkatoh@ncc.go.jp

Key words: bioinformatics, comparative genomics, comparative proteomics, Angiopoietin, WNT, integrome network, systems medicine

\section{Introduction}

Angiogenesis is regulated by the balance between pro-angiogenic factors (angiogenic activators) and anti-angiogenic factors (angiogenic inhibitors) (1-6). Angiopoietin-1 (ANGPT1), Angiopoietin-4 (ANGPT4), VEGF, FGF2, FGF4, HGF, Ephrin, IL8 and CXCL12 (SFD1) are pro-angiogenic factors, while Angiopoietin-2 (ANGPT2), Angiostatin, Endostatin, Tumstatin, Canstatin, THBS1, THBS2, TNFSF15 (VEGI) and Vasohibin (VASH1) are anti-angiogenic factors (1-17).

ANGPT1, ANGPT2, ANGPT4 are ligands for TIE family receptor tyrosine kinases, TIE1 and TIE2 (TEK) (7-9). ANGPTL1 (ANGPT3), ANGPTL2, ANGPTL3 (ANGPT5), ANGPTL4, ANGPTL5, ANGPTL6 and ANGPTL7 (18-23) are related to ANGPT1, ANGPT2 and ANGPT4. Angiopoietin family consists of ANGPT1, ANGPT2, ANGPT4, ANGPTL1, ANGPTL2, ANGPTL3, ANGPTL4, ANGPTL5, ANGPTL6 and ANGPTL7.

WNT, FGF, Notch and Hedgehog signaling pathways network together during embryogenesis, tissue regeneration and carcinogenesis (24-33). Canonical WNT signals are transduced to the transcriptional complex consisting of TCF/LEF, ß-catenin, BCL9/BCL9L and PYGO1/PYGO2 to activate transcription of target genes, such as DKK1, DKK4, FGF 18 and FGF20 (34-43); however, WNT-dependent transcriptional regulation of Angiopoietin family members remains unclear.

Here, TCF/LEF binding sites within the promoter region of human Angiopoietin family members were searched for by using bioinformatics and human intelligence (Humint). Because four TCF/LEF-binding sites were identified in the 5'-promoter region of human ANGPTL7 gene, comparative genomics analyses on ANGPTL7 orthologs were further performed.

\section{Materials and methods}

WNT target gene screening. Genome sequences corresponding to human ANGPT1, ANGPT2, ANGPT4, ANGPTL1, ANGPTL2, ANGPTL3, ANGPTL4, ANGPTL5, ANGPTL6 and ANGPTL7 genes were searched for with BLAST programs (http://www.ncbi.nlm.nih.gov) as described previously (44-47). TCF/LEF-binding sites within the 5'-flanking promoter region of the above genes were searched for based on bioinformatics and manual inspection as described previously $(38-42,48)$.

Identification of chimpanzee and cow ANGPTL7 orthologs. Chimpanzee and cow genome sequences homologous to 
A

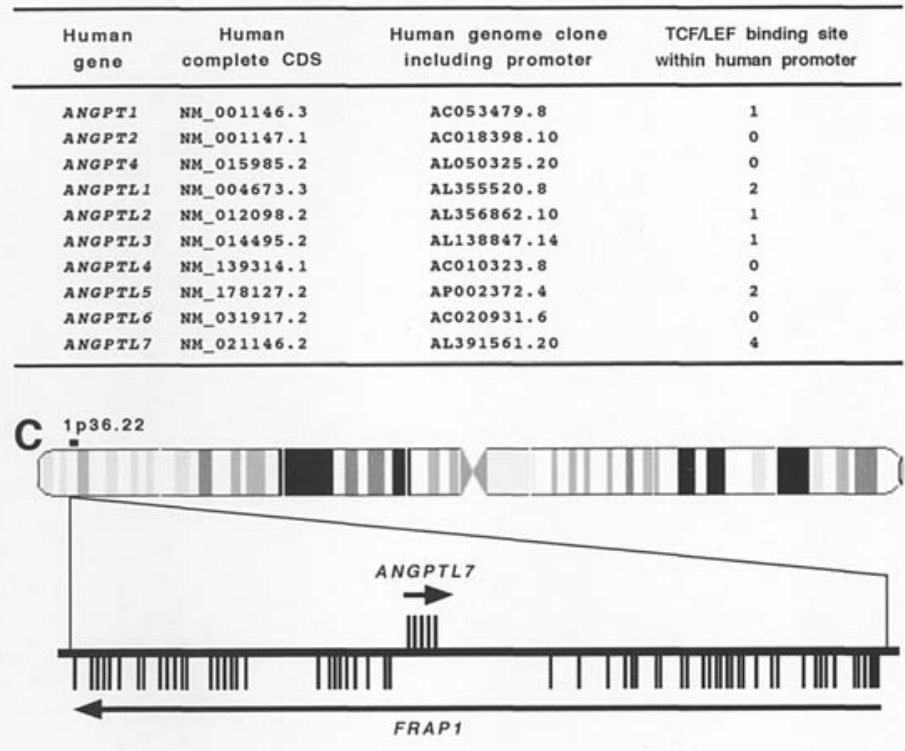

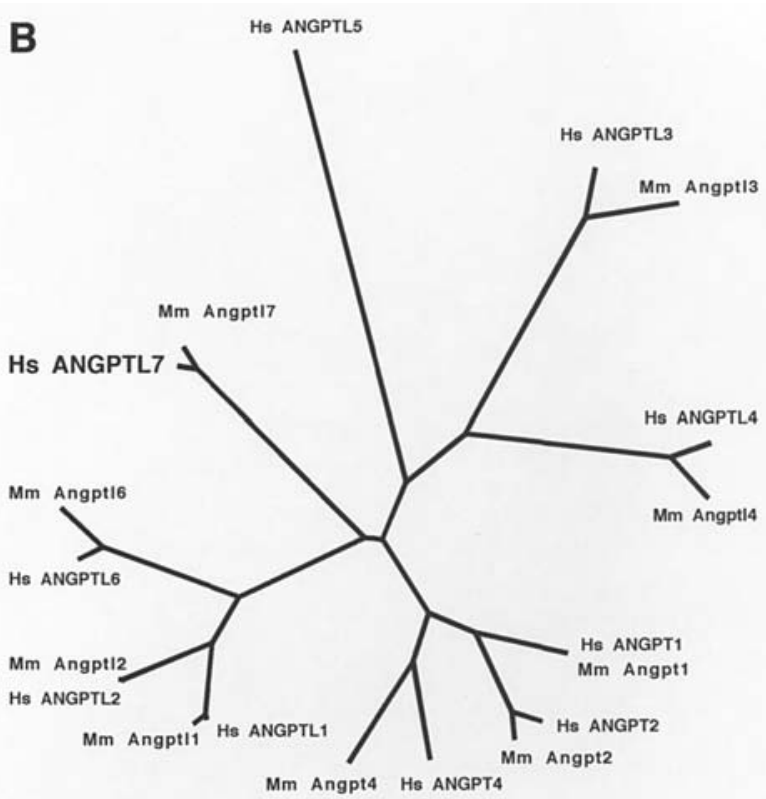

Figure 1. (A), Human Angiopoietin gene family. Gene symbol, complete coding sequence, genome sequence and the number of TCF/LEF-binding sites within promoter region of Angiopoietin family genes are listed. Four TCF/LEF-binding sites exist within the human ANGPTL7 promoter. (B), Phylogenetic analysis on human and mouse Angiopoietin family members. Hs, human; Mm, mouse. (C), ANGPTL7 locus at human chromosome 1p36.22. ANGPTL7 gene, consisting of five exons, is located within intron 28 of the FRAPl gene.

human ANGPTL7 were searched for with BLAST programs as described previously (49-52). TCF/LEF-binding sites within the 5'-flanking promoter region of ANGPTL7 orthologs were also searched for.

Comparative proteomics analysis. Phylogenetic analysis on ANGPT family proteins was performed by using the CLUSTALW program.

Comparative genomics analyses. Phylogenetic analysis on promoter of ANGPTL7 orthologs was performed by using the CLUSTALW program. Promoter region of human and chimpanzee ANGPTL7 orthologs were aligned by using the Genetyx program and manual curation as described previously (53-56).

In silico expression analyses. Expressed sequence tags (ESTs) derived from human ANGPTL7 gene and mouse Angptl7 gene were searched for by using the BLAST programs. The sources of human ANGPTL7 ESTs and those of mouse Angpt17 ESTs were listed up for in silico expression analyses.

\section{Results}

Screening of the TCF/LEF-binding site within promoter region of Angiopoietin family genes. Human ANGPT1 RefSeq (NM_001146.3), ANGPT2 RefSeq (NM_001147.1), ANGPT4 RefSeq (NM_015985.2), ANGPTL1 RefSeq (NM_004673.3), ANGPTL2 RefSeq (NM_012098.2), ANGPTL3 RefSeq (NM_014495.2), ANGPTL4 RefSeq (NM_139314.1), ANGPTL5 RefSeq (NM_178127.2), ANGPTL6 RefSeq (NM_031917.2) and ANGPTL7 RefSeq (NM_021146.2) were used as query sequences for the BLAST programs to identify genome clones corresponding to Angiopoietin family genes. The 5'-flanking promoter region of human ANGPT1,
ANGPT2, ANGPT4, ANGPTL1, ANGPTL2, ANGPTL3, ANGPTL4, ANGPTL5, ANGPTL6 and ANGPTL7 genes were identified within AC053479.8, AC018398.10, AL050325.20, AL355520.8, AL356862.10, AL138847.14, AC010323.8, AP002372.4, AC020931.6 and AL391561.20 genome sequences, respectively (Fig. 1A). TCF/LEF-binding sites within the 5'-promoter region of human Angiopoietin family genes were then searched for based on manual inspection. Four TCF/LEF-binding sites were identified within human ANGPTL7 promoter (Fig. 1A).

Identification of chimpanzee ANGPTL7 ortholog. BLAST programs using human ANGPTL7 RefSeq revealed that chimpanzee ANGPTL7 gene was located within NW_101546.1 genome sequence (Fig. 2A). Exon-intron boundaries of the chimpanzee ANGPTL7 gene were determined based on the consensus sequence of exon-intron junctions. Chimpanzee ANGPTL7 gene was found consisting of five exons.

Compared with human ANGPTL7 RefSeq, one-base insertion occurred at exon 3 of chimpanzee ANGPTL7 gene within NW_101546.1 genome sequence. Re-sequencing of the genome sequence around exon 3 of chimpanzee ANGPTL7 gene should be done in the future to correct the sequencing error.

Complete coding sequence (CDS) of chimpanzee ANGPTL7 was determined in this study by assembling nucleotide sequences of five exons (Fig. 2A). Genetyx program revealed that nucleotide position 241-1281 was the coding region of chimpanzee ANGPTL7 complete CDS. Chimpanzee ANGPTL7 gene was found to encode a 346-amino-acid ANGPTL7 protein (Fig. 2A).

Comparative proteomics analysis on ANGPTL7 orthologs. Phylogenetic analysis on human and mouse Angiopoietin family members revealed that ANGPTL7 orthologs were 


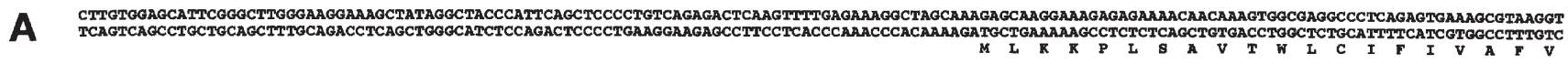
AGCCACCCAGCTGGCTGCAGAAGCTCTCTAAGCGCAAGACACCAGCACAGCCACAGCTCAAAGCGGCCAACTGCTGTGAGGAGGTGAaGGAGCTCAAGGCCCAAGTTGCCAACCTTAGCAGCCTGCTGAGTGAACTGAACAAGAAGCAG GAGAGGACTGGGTCAGGGTGTCATGCAGGTATGGAGCTGGAGAGCAACAGCAAGCGCATGGAGTCCGGCTCACAGATGCTGAGAGCAAGTACTCCGAGATGAACAACCAAATCGACATCATOCAGCTGCAGGCAGCGCAGACGGTC

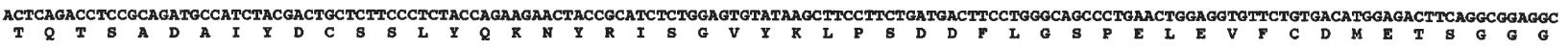
TGGACCATCATCCAGAGACGAAAAAGTGGCCTTGTCTCCTTCTACCGGGACTGGAAGCAGTACAAGCAGGGCTFTGGCAGCATCCGTGGGGCTTCTGGCTGGGAACGAACACATCCACCGGCTCTCCAGACAGCCAACCCCGCCTGCGT GTAGAGATGGAGGACTGGAGGGCAACCTGCCCTACGCTGAGTATAGCCACTPTGTTTTGGGCAATGACTCAACAGCTATCGCCTCTTCCTGGGGACTACACTGGCAATGTGGGGACGACGCCCTCCAGTATCATAACAACACAGCC TTCACCACCAAGGACAAGGACAATGACAACTGCTTGGACAAGTGTGCACAGCTCCCAAAGGTGGCTACTGGTACAACTGCTGCACAGACTCCAACCTCAATGGAGTGTACTACCGCCTGGGTGAGCACAATAAGCACCTGGATGGCATC ACCTGGTACGGCTGGCATGGATCTACCTACTCCCTCAAACGGGTGGAGATGAAAATCCGCCCGGAAGACTTCAAGCCTTAAAAGGAGGCTGCCGTGGAGCACGGATACAGAAACTGAGACACGTGGAGACTGGATGAGGGCAGATGAGGA CAGGAAGAGAGTGTTAGAAAGGGTAGGACTGAGAAACAGCCTATAATCTCCAAAGAAAGAATAAGTCTCCAAGGAGCACAAAAAAATCATATGTACCAAGGATGTTACAGTAAACAGGATGAACTATTTAAACCCACTGGGTCCTGCCAC

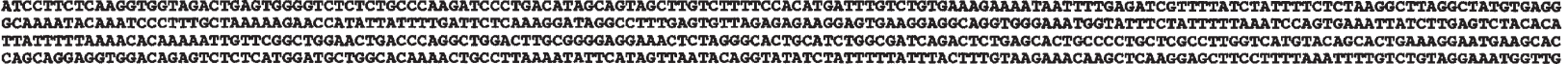

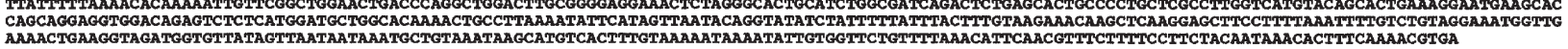

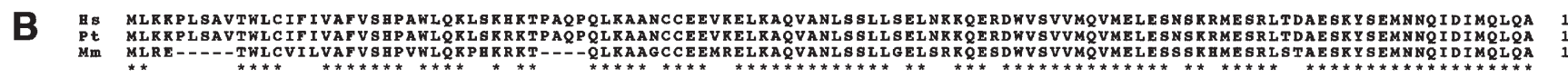

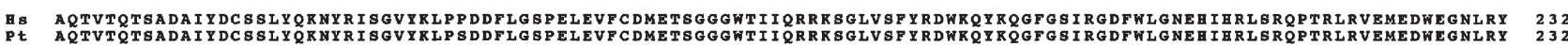

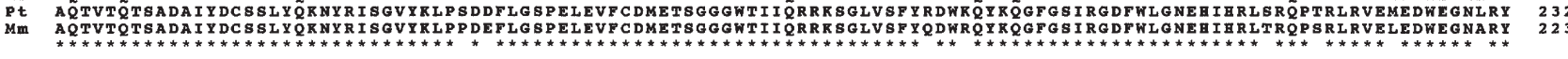

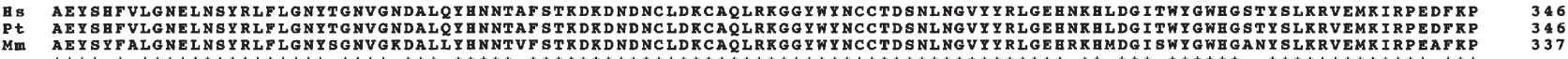

Figure 2. Chimpanzee ANGPTL7. (A), Nucleotide and amino-acid sequences of chimpanzee ANGPTL7 complete CDS. Nucleotides and amino-acid residues are numbered on the right. (B), Alignment of ANGPTL7 orthologs. Hs, human; Pt, chimpanzee; Mm, mouse. Conserved amino-acid residues are shown by asterisks.

Hs CTCAACAGAATCTTTTGATTAGAATGCCAAGTTGCTATAAGGGTATCAGTAACATCCTCACCCTTTAGCAGGCAAAGGAGGTGGGTATGTGAGAAAACAAACTCTGATCCACCTGATCCTCCACTGCTTTTTGATTCTGAGAAAAAAA CTCAACAGAATCTTTTGATTAGAATGCAAGTTGCTATAAGGGTATCAGTAACATCCTCACCCTTTAGCAGGCAAAGGAGGCTGGTATGTGAGAAAACAAACTCTGATCCACTGATCCTCTACTGCTITTTGATTCTGAGAAAAAAAAA S AAATTGTTCAGTAACTCCAGCTGCCTGAACAGAAATTACTTTCTGAGGAGCTAGAAGCCCTGACTGGAAAAGAGCTCTGGGGGATTATGGCATGGGAGGAGAGCTGTCAGGCATCAGGCTTATGTGGGAGGACCCACGCTGACTACAAC

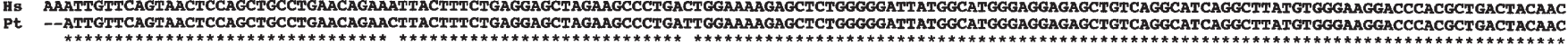
TCF/LEF

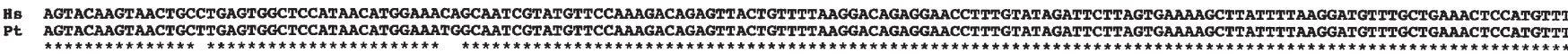
$\underset{T C F / L E F}{====x=}$

Hs TTCACATTTCTTTTCAAAAGAGCATTGTCCTTCATATATAGTACATGAAAAATTACAATATTCATGTTTTTTIAGATACAAAGTCATATACAAATAGAGGAGGAATAATTTCAAATTGCAAACCATACCAGCAAAGAAAGAGGGAAATGAC

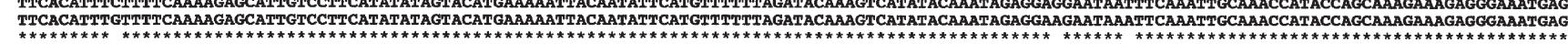
Hs ACTCTAGATATGGATTPATTAGGTGACAGGGATTCTPTTCTCTATATTPTAATATATTTTCCAAGTTTTGAACCACGGGCTAAGGATGGCCTGTTCTCTGTCCTGCCTAGAAACTGACAGAAGCATGGGTGACCAATCCCAGACATGCT TCF/LEF

TCF/LEF

H TGGCACTGCTGAGTTTTCATCTCTGTTGTAATCTTCTGACACCTCCTTGCAAAATATCAAGTGTTATATGCTAATACTGGCCCTCTCTTTGTTGAAGGAGAAAGCATGTAAAAAGACAACTGTCCAAACCCTGTGGCTACAAAGTCTTT

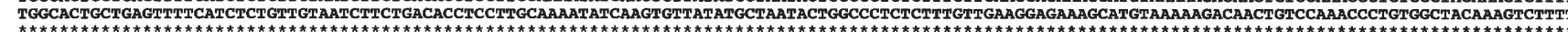
H TTTGGCTTCCTAAGAGATTTACTCTATTCTTCTATTTCTAGAACTTTTCTAAGTTGAAGATTTCAAATGTGAGATAGTGTTAGATCCAGGTTGAGTAAGGATTTTATAAAATACTAAATAATGATCAGAGATGGGATAATCAGCCTAG

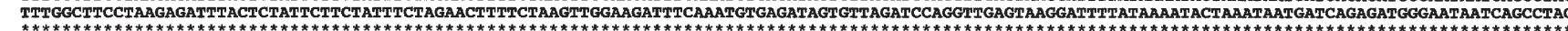
H ATAAAAGCATATTCAGCAGGATGTCAAAATATACCAGATCCTCATATAAACAGCATGGATTACATTAACAGGCCTCCCTGGGTGAGTAGCGTCTCTTTCGCCTACCTTACGCTCACCCCATGGTTATATAACCTTTTGAACGGAATTTGG ATAAAAGCATATTCAGCAGGATGTCAAAATATACCAGATCCTCATATAAACAGCATGGATTACATTAACAGGCCTCCCTGGGTGAGTACGTCTCTTTTGCCTACCTTACGCTCACCCATGGTTATATAACCTYTTGAACGGAATPGG

Hs AAATTCTTCTGACCCTGTCCTGCTTGCTGATAGAGTTGAGCCAGTGGCTGGCAGGGGATAATCTAATAGGCGAAACTGGAAACACACAAATTCACAGTCCTCTTGTCTTAGCCCAGAGACTGTACAGGCAAGGAAAGAAAGACTCGCCC AAATTCCTCTGACCCTGCCCTGCTTGCTGATAGAGTTGAGCCAGTGGCTGGCAGGGCATAATCTAATAGGCGAAATGGAAACACACAAATTCACAGTCCTCTTTTCTCAGCCCAGAGACTGTACAGGCAAGGAAAGAAAGACTCGCCC

Hs CATCTCCCTCCTCCCCTCCTCTGGCCTAAGTTGCCGCTGACTTCACCCAACAGGCACCTGACCCTCCCAGATGAGCTGGGAGGGGCTAAAGCCTGGT C CAGCCATGGTAGGGGTGGAGGTACAGGCAGCAAACAATATTTAAGATGCTGA CATCTCCCTCCTCCCCTCCTCTGGCCTAAGTGCCGCTGACTTCACCCAACAGGCACCTGACCCTCCCAGATGAGCTGGGAGGGCTAAAGCCTGGTGCAGCCATGGTAGGGTGGAGGTACAGGCAGCAAACAATATTTAAGATGCTGA

Figure 3. Alignment of the human and chimpanzee ANGPTL7 promoters. Hs, human; Pt, chimpanzee. Region corresponding to exon 1 of the human ANGPTL7 gene is boxed. TCF/LEF-binding sites are shown by double over-lines.

relatively well conserved (Fig. 1B). Chimpanzee ANGPTL7 showed $99.4 \%$ and $86.1 \%$ total-amino-acid identity with human ANGPTL7 and mouse Angpt17, respectively (Fig. 2B).

Intra-species comparative genomics analysis on ANGPTL7 orthologs. ANGPTL7 gene was located within FRAP1 gene at human chromosome 1p36.22 (Fig. 1C). Paralog of FRAP1 gene, encoding mTOR protein, was not identified within the human genome. ANGPTL7 gene was found consisting of 5 exons, while FRAP1 gene was found consisting of 58 exons.
ANGPTL7 gene was located within intron 28 of FRAP1 gene in the anti-sense direction (Fig. 1C). These facts indicate that $A N G P T 7 L$ gene was inserted into the FRAPl gene during evolution.

Expression profile of human ANGPTL7 and mouse Angptl7 $m R N A s$. In silico expression analyses were performed to compare the expression profiles of human ANGPTL7 and mouse Angptl7 mRNAs. Human ANGPTL7 mRNA was expressed in neural tissues, keratoconus cornea, trabecular 
meshwork, melanotic melanoma and uterus endometrial cancer, while mouse Angptl7 mRNA was expressed in four-cell embryo, synovial fibroblasts, thymus, uterus and testis.

Comparative genomics analyses on ANGPTL7 promoters. Human and chimpanzee ANGPTL7 promoters were located within AL391561.20 and NW_101546.1 genome sequences, respectively, as mentioned above. BLAST programs revealed that cow, mouse and rat Angptl7 promoters were located within AC174033.2, AC108508.2 and AC125863.3 genome sequences, respectively. Cow Angptl7 promoter was not used for the following comparative genomics analyses, because one of sequencing gaps within the AC174033.2 genome sequence corresponded to the cow Angptl7 promoter region.

GC contents of human, chimpanzee, mouse and rat ANGPTL7 promoters were $41.5 \%, 41.4 \%, 50.2 \%$ and $50.6 \%$, respectively. GC contents of primate ANGPTL7 promoters were lower than those of rodent Angptl7 promoters.

Four TCF/LEF-binding sites within human VEGFD promoter were located about 1050, 900, 600, and 550 bp upstream of the transcription start site (Fig. 3). Four TCF/ LEF-binding sites within the human ANGPTL7 promoter were conserved in chimpanzee ANGPTL7 promoter; however, only an unrelated TCF/LEF-binding site occurred in the mouse and rat Angptl7 promoters.

\section{Discussion}

TCF/LEF binding sites within the promoter region of human ANGPT1, ANGPT2, ANGPT4, ANGPTL1, ANGPTL2, ANGPTL3, ANGPTL4, ANGPTL5, ANGPTL6 and ANGPTL7 genes were searched for in this study. Because four TCF/ LEF-binding sites were identified within the human ANGPTL7 promoter (Fig. 1A), comparative genomics analyses on ANGPTL7 orthologs were further performed.

ANGPTL7 gene at human chromosome 1p36.22 was located within intron 28 of the FRAP1 gene encoding mTOR protein (Fig. 1C). Chimpanzee ANGPTL7 gene, consisting of five exons, was located within the NW_101546.1 genome sequence (Fig. 2A). Chimpanzee ANGPTL7 showed 99.4\% and $86.1 \%$ total-amino-acid identity with human ANGPTL7 and mouse Angpt17, respectively (Fig. 2B).

Four TCF/LEF-binding sites within the human ANGPTL7 promoter were conserved in chimpanzee ANGPTL7 promoter (Fig. 3); however, only an unrelated TCF/LEF-binding site occurred in the mouse and rat Angptl7 promoters. GC contents of primate $A N G P T L 7$ promoters were lower than those of rodent Angptl7 promoters. Human ANGPTL7 mRNA was expressed in neural tissues, keratoconus cornea, trabecular meshwork, melanotic melanoma and uterus endometrial cancer, while mouse Angptl7 mRNA was expressed in four-cell embryo, synovial fibroblasts, thymus, uterus and testis. Together these facts indicate that expression profiles of primate ANGPTL7 orthologs and rodent Angptl7 orthologs differ due to promoter evolution.

Human ANGPTL7, characterized as a potent target gene of $\mathrm{WNT} / ß$-catenin signaling pathway, is a pharmacogenomics target in the fields of oncology and regenerative medicine.

\section{References}

1. Folkman J: Angiogenesis in cancer, vascular, rheumatoid and other disease. Nat Med 1: 27-31, 1995.

2. Hanahan D and Folkman J: Patterns and emerging mechanisms of the angiogenic switch during tumorigenesis. Cell 86: 353-364, 1996.

3. Kerbel RS and Folkman J: Clinical translation of angiogenesis inhibitors. Nat Rev Cancer 2: 727-739, 2002.

4. Carmeliet P: Angiogenesis in life, disease and medicine. Nature 438: 932-936, 2005 .

5. Coultas L, Chawengsaksophak K and Rossant J: Endothelial cells and VEGF in vascular development. Nature 438: 937-945, 2005.

6. Kerbel RS: Vasohibin: the feedback on a new inhibitor of angiogenesis. J Clin Invest 114: 884-886, 2004

7. Suri C, Jones PF, Patan S, et al: Requisite role of angiopoietin-1, a ligand for the TIE2 receptor, during embryonic angiogenesis. Cell 87: 1171-1180, 1996.

8. Maisonpierre PC, Suri C, Jones PF, et al: Angiopoietin-2, a natural antagonist for Tie2 that disrupts in vivo angiogenesis. Science 277: 55-60, 1997.

9. Valenzuela DM, Griffiths JA, Rojas J, et al: Angiopoietins 3 and 4: diverging gene counterparts in mice and humans. Proc Natl Acad Sci USA 96: 1904-1909, 1999.

10. Tammela T, Enholm B, Alitalo K and Paavonen K: The biology of vascular endothelial growth factors. Cardiovasc Res 65: 550-563, 2005.

11. Schweigerer L, Neufeld G, Friedman J, et al: Capillary endothelial cells express basic fibroblast growth factor, a mitogen that promotes their own growth. Nature 325: 257-259, 1987.

12. Zugmaier G, Lippman ME and Wellstein A: Inhibition by pentosan polysulfate (PPS) of heparin-binding growth factors released from tumor cells and blockage by PPS of tumor growth in animals. J Natl Cancer Inst 84: 1716-1724, 1992.

13. Rasmussen HS, Rasmussen CS, Macko J and Yonehiro G: Angiogenic gene therapy strategies for the treatment of cardiovascular disease. Curr Opin Mol Ther 4: 476-481, 2002.

14. Langer R, Conn H, Vacanti J, Haudenschild C and Folkman J: Control of tumor growth in animals by infusion of an angiogenesis inhibitor. Proc Natl Acad Sci USA 77: 4331-4335, 1980.

15. Kalluri R: Discovery of type IV collagen non-collagenous domains as novel integrin ligands and endogenous inhibitors of angiogenesis. Cold Spring Harb Symp Quant Biol 67: 255-266, 2002 .

16. Bocci G, Francia G, Man S, Lawler J and Kerbel RS: Thrombospondin-1, a mediator of the anti-angiogenic effects of low-dose metronomic chemotherapy. Proc Natl Acad Sci USA 100: 12917-12922, 2003.

17. Watanabe K, Hasegawa Y, Yamashita H, et al: Vasohibin as an endothelium-derived negative feedback regulator of angiogenesis. J Clin Invest 114: 898-907, 2004.

18. Kim I, Moon SO, Koh KN, et al: Molecular cloning, expression, and characterization of angiopoietin-related protein. Angiopoietinrelated protein induces endothelial cell sprouting. J Biol Chem 274: 26523-26528, 1999.

19. Conklin D, Gilbertson D, Taft DW, et al: Identification of a mammalian angiopoietin-related protein expressed specifically in liver. Genomics 62: 477-482, 1999.

20. Yoon JC, Chickering TW, Rosen ED, et al: Peroxisome proliferator-activated receptor $\gamma$ target gene encoding a novel angiopoietin-related protein associated with adipose differentiation. Mol Cell Biol 20: 5343-5349, 2000.

21. Zeng L, Dai J, Ying K, et al: Identification of a novel human angiopoietin-like gene expressed mainly in heart. J Hum Genet 48: 159-162, 2003.

22. Oike Y, Yasunaga K, Ito Y, et al: Angiopoietin-related growth factor (AGF) promotes epidermal proliferation, remodeling, and regeneration. Proc Natl Acad Sci USA 100: 9494-9499, 2003.

23. Peek R, Kammerer RA, Frank S, Otte-Holler I and Westphal JR: The angiopoietin-like factor cornea-derived transcript 6 is a putative morphogen for human cornea. J Biol Chem 277: 686-693, 2002.

24. Katoh M: WNT and FGF gene clusters. Int J Oncol 21: 1269-1273, 2002.

25. Katoh M: Epithelial-mesenchymal transition in gastric cancer. Int J Oncol 27: 1677-1683, 2005

26. Katoh $Y$ and Katoh M: FGF signaling inhibitor, SPRY4, is evolutionarily conserved target of WNT signaling pathway in progenitor cells. Int J Mol Med 17: 529-532, 2006. 
27. Li JL and Harris AL: Notch signaling from tumor cells: a new mechanism of angiogenesis. Cancer Cell 8: 1-3, 2005.

28. Katoh M and Katoh M: Notch ligand, JAG1, is evolutionarily conserved target of canonical WNT signaling pathway in progenitor cells. Int J Mol Med 17: 681-685, 2006.

29. Lawson ND, Vogel AM and Weinstein BM: Sonic hedgehog and vascular endothelium growth factor act upstream of Notch pathway during arterial endothelial differentiation. Dev Cell 3: 127-136, 2002.

30. Katoh Y and Katoh M: Comparative genomics on HHIP family orthologs. Int J Mol Med 17: 391-395, 2006.

31. Katoh $\mathrm{Y}$ and Katoh M: Hedgehog signaling in gastric cancer. Cancer Biol Ther 4: 1050-1054, 2005.

32. Garciadiego-Cazares D, Rosales C, Katoh M and ChimalMonroy J: Coordination of chondrocyte differentiation and joint formation by $\alpha 5 \beta 1$ integrin in the developing appendicular skeleton. Development 131: 4735-4742, 2004.

33. Katoh Y and Katoh M: WNT antagonist, SFRP1, is Hedgehog signaling target. Int J Mol Med 17: 171-175, 2006.

34. Katoh M: Regulation of WNT signaling molecules by retinoic acid during neuronal differentiation in NT2 cells: threshold model of WNT action. Int J Mol Med 10: 683-687, 2002.

35. Katoh $M$ and Katoh M: Identification and characterization of human $B C L 9 L$ gene and mouse Bcl9l gene in silico. Int J Mol Med 12: 643-649, 2003.

36. Heller RS, Klein T, Ling Z, Heimberg H, Katoh M, Madsen OD and Serup P: Expression of WNT, Frizzled, sFRP, and DKK genes in adult human pancreas. Gene Expr 11: 141-147, 2003.

37. Swain RK, Katoh M, Medina A and Steinbeisser H: Xenopus frizzled-4S, a splicing variant of Xfz4, is a context-dependent activator and inhibitor of Wnt/ß-catenin signaling. Cell Commun Signal 3: 12, 2005.

38. Katoh Y and Katoh M: Comparative genomics on $D K K 1$ orthologs. Int J Oncol 27: 275-279, 2005.

39. Katoh Y and Katoh M: Comparative genomics on $D K K 2$ and DKK4 orthologs. Int J Mol Med 16: 477-481, 2005.

40. Katoh Y and Katoh M: Comparative genomics on FGF16 orthologs. Int J Mol Med 16: 959-963, 2005.
41. Katoh $\mathrm{M}$ and Katoh M: Comparative genomics on $F G F 8$, $F G F 17$, and $F G F 18$ orthologs. Int J Mol Med 16: 493-496, 2005.

42. Katoh $\mathrm{M}$ and Katoh M: Comparative genomics on FGF20 orthologs. Oncol Rep 14: 287-290, 2005.

43. Katoh M: WNT2B: comparative integromics and clinical application. Int J Mol Med 16: 1103-1108, 2005.

44. Katoh M: Paradigm shift in gene-finding method: from benchtop approach to desk-top approach. Int J Mol Med 10: 677-682, 2002.

45. Katoh $\mathrm{M}$ and Katoh $\mathrm{M}$ : Identification and characterization of human HES2, HES3, and HES5 genes in silico. Int J Oncol 25: 529-534, 2004.

46. Katoh M and Katoh M: Identification and characterization of human HESL, rat Hesl and rainbow trout hesl genes in silico. Int J Mol Med 14: 747-751, 2005.

47. Katoh $\mathrm{Y}$ and Katoh M: Identification and characterization of rat Wnt6 and Wnt10a genes in silico. Int J Mol Med 15: 527-531, 2005.

48. Katoh Y and Katoh M: Comparative genomics on SLIT1, SLIT2, and SLIT3 orthologs. Oncol Rep 14: 1351-1355, 2005.

49. Katoh $\mathrm{Y}$ and Katoh M: Identification and characterization of rat Wnt1 and Wnt10b genes in silico. Int J Oncol 26: 841-845, 2005.

50. Katoh $\mathrm{M}$ and Katoh $\mathrm{M}$ : Comparative genomics on WNT8A and $W N T 8 B$ genes. Int J Oncol 26: 1129-1133, 2005.

51. Katoh M: Molecular evolution of WNT2B orthologs. Int J Oncol 26: 1135-1139, 2005

52. Katoh M: Comparative genomics on WNT3-WNT9B gene cluster. Int J Mol Med 15: 743-747, 2005.

53. Katoh $\mathrm{M}$ and Katoh M: Comparative genomics on WNT5A and WNT5B genes. Int J Mol Med 15: 749-753, 2005.

54. Katoh Y and Katoh M: Comparative genomics on WNT11 gene. Int J Mol Med 15: 879-883, 2005.

55. Katoh Y and Katoh M: Comparative genomics on VANGL1 and VANGL2 genes. Int J Oncol 26: 1435-1440, 2005.

56. Katoh Y and Katoh M: Comparative genomics on SFRP1 orthologs. Int J Oncol 27: 861-865, 2005. 